- the papers whose authors declined, as measured by downloads to a free online reference manager, Mendeley. The Laura and John Arnold Foundation, based in Houston, Texas, says that it is actively considering funding Science Exchange to validate cancer-cell biology papers within Iorns' cohort.

Some at the NIH are coming round to the idea that validation is best contracted out. Shai Silberberg, who is responsible for reproducibility issues at the agency's neurology institute, has almost finished a pilot study in which several academic labs tried to reproduce findings from studies aiming to move drugs to a stage at which they are ready to be tested in humans. He points out that it has already taken two and a half years. "It's too slow," he says. He now favours speedier contract-research organizations.

Iorns, for her part, is not waiting for the $\mathrm{NIH}$ to take action. On 30 July, Science Exchange launched a programme with reagent supplier antibodies-online.com, based in Aachen, Germany, to independently validate research antibodies. These are used, for example, to probe gene function in biomedical experiments, but their effects are notoriously variable. "Having a third party validate every batch would be a fabulous thing," says Peter Park, a computational biologist at Harvard Medical School. He notes that the consortium behind ENCODE - a project aimed at identifying all the functional elements in the human genome - tested more than 200 antibodies targeting modifications to proteins called histones and found that more than $25 \%$ failed to target the advertised modification.

With antibodies, the companies that make them have an incentive to prove the quality of their products, and Iorns hopes that they will pay the thousands of dollars that such validation costs. Antibodies that pass muster will receive an 'independently validated' green tick in the antibodiesonline.com catalogue.

But with budgets stretched thin - and with Congress well aware of the reproducibility issues - the NIH also has an incentive to make sure that its $\$ 29$-billion budget is spent on verifiable science. "We are obligated to consider how we want to address this," says Tabak. .

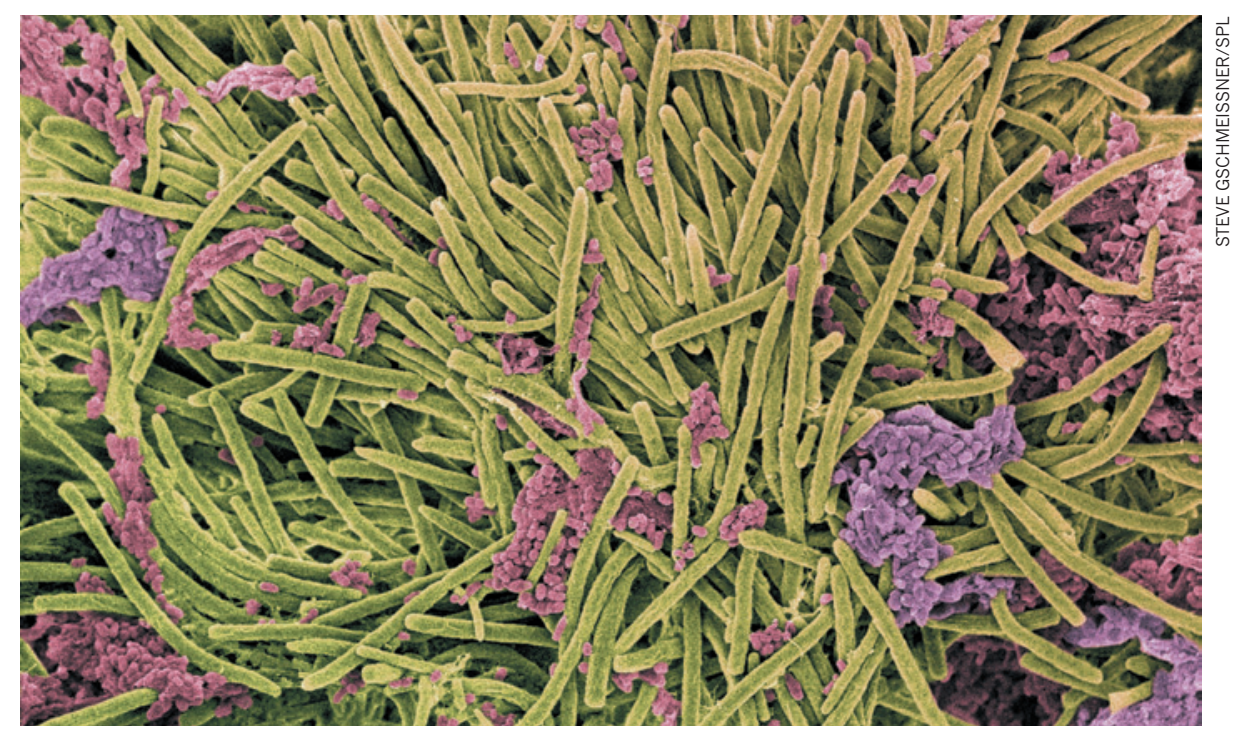

The tongue is one of many sites to have its bacteria catalogued by the Human Microbiome Project.

\title{
MICROBIOLOGY
}

\section{Microbiome research goes without a home}

\section{Scientists say core tools and expertise remain necessary.}

\section{BY BETH MOLE}

$\mathrm{T}$ Trillions of microorganisms call the human body home. But 'home' for many US scientists studying these microscopic squatters is about to change, as funding for human microbiome research scatters across 16 of the 27 centres of the US National Institutes of Health (NIH).

Last year, researchers completed the \$US173-million Human Microbiome Project, which took five years and generated a slew of reference data, mostly genetic sequences of all the microbes that dwell on and inside humans. But the project's scientists fear that a lack of standards and expertise in data-gathering and analysis are hampering efforts to extract meaning from this information.

At a meeting last week in Bethesda, Maryland, they reiterated that identifying the microbes is just the first step. Researchers must also focus on how bacteria interact with each other and the human body to cause - or prevent - disease. Yet these calls for action are coming as the project faces significant downsizing: by the beginning of 2014, microbiome researchers will no longer be able to depend on centralized resources based at the NIH's National Human Genome Research Institute (NHGRI) in Bethesda.

"The microbiome has so much appeal," says Christian Jobin, an immunologist at the University of Florida in Gainesville, who studies the interplay between gut microbes, inflammation and cancer. "But we're lacking direction right now."

In 2012, the project culminated with a flurry of publications (D. A. Relman Nature 486, 194195; 2012). But, says Lita Proctor, the project's programme director, some efforts still seem too

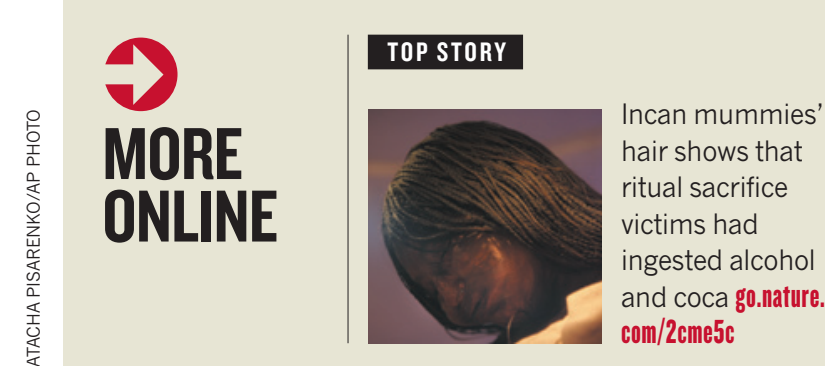

\section{MORE NEWS}

- Pilot projects bury carbon dioxide in porous basalt layers go.nature.com/zop2ew - Conflicting studies rekindle debate on why monogamy evolved in primates go.nature.com/9efoog - An artificial-intelligence algorithm for quantum computers go.nature.com/vto8pf

\section{NATURE PODCAST}

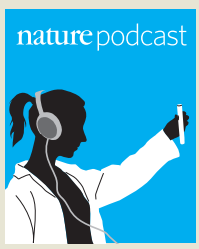

Lactose tolerance in ancient Europeans, nanodiamonds inside cells, and Middle East science nature.com/nature/ podcast 
focused on data-gathering and do not account for the complex ways in which microbes interact with one another and their hosts. Some scientists are "struggling to figure out how to think about the microbiome", she says.

There are several problems, says Rob Knight, a microbial ecologist at the University of Colorado Boulder. First, scientists need to forge standards on matters such as how long to wait after a person has a shower before swabbing their skin for microbes, or what a person ate before collecting a stool sample. Although researchers who gathered the reference data sets from healthy individuals tried to establish such standards, some of these have been ignored, and there is also wide variation in the way that microbiome samples are amassed from people with diseases.

Second, collecting, sequencing and analysing DNA from thousands of microbial species living in and on humans requires an interdisciplinary team with knowledge of clinical ethics, engineering and bioinformatics - expertise that can be tricky to assemble.

Last, it remains difficult to establish whether a microbial trend associated with a disease is the cause or a result of that disease. One solution, Knight says, would be to track people over time, to allow researchers to detect microbial changes that occur before or after someone becomes ill. Soon, he hopes, microbiome information could help doctors to predict a patient's risk of developing various diseases, and conditions such as obesity.

Jobin says few microbiologists are trying to understand what the microbes are doing and how they might be controlled. This year, he

"Scientists are sat on a review panel Scientists are for grant applications struggling to figure out how to think about the microbiome." proposing to explore the microbiome's connection to gastrointestinal cancers, and noted that many planned simply to sequence microbial communities. They were swiftly rejected. "There are very few mechanism-driven studies," he says.

Expectations are higher now than they were in 2003, when the Human Genome Project wrapped up, says Owen White, a bioinformatics researcher at the University of Maryland School of Medicine in Baltimore. "When you were sequencing the human genome, or the next mammalian genome, everyone knew that that was relatively hypothesis-free - and that was fine," he says.

The Human Genome Project was overseen by the NHGRI, which continues to lead genomerelated work. By contrast, the Human Microbiome Project does not have a place to call home for its second phase. Over its first five years, it received $\$ 146$ million from the NIH common fund, money that was managed by the NHGRI and that contributed to the development of field-wide tools. But in its second phase, from 2014 to 2016 , only $\$ 15$ million in common-fund money will flow through the NHGRI. Microbiome research will instead be largely supported by 16 individual NIH institutes.

The project's leaders say that the effort still needs a base to provide resources such as standardized sampling protocols, technical support, and microbiome samples and data from specific patient groups that researchers can mine. Many had assumed that the NHGRI would take on this role, because the microbiome project was initially seen as an extension of the Human Genome Project. But the NHGRI "has been less enthusiastic than expected”, Knight says. Jane Peterson, a senior adviser to the NHGRI director's office, says that the future of human microbiome research will be in clinical applications, which does not fit with the NHGRI's mission.

But Heidi Kong, a dermatologist and microbiome researcher at the National Cancer Institute in Bethesda, says that the project's basic science needs to be nurtured further before it will be ready for the clinic. Scientists need first to pin down the function of individual microbes and the body's response to them, and should only then begin testing treatments on those interactions. "There is a bit more work we need to do," Kong says. 